\title{
Genetic diversity of local Yunnan chicken breeds and their relationships with Red Junglefowl
}

\author{
J.L. Huo ${ }^{1 *}$, G.S. Wu ${ }^{2 *}$, T. Chen ${ }^{3 *}$, H.L. Huo ${ }^{4}$, F. Yuan ${ }^{1}$, L.X. Liu ${ }^{4}$, \\ C.R. Ge ${ }^{1}$ and Y.W. Miao ${ }^{1}$
}

${ }^{1}$ Faculty of Animal Science and Technology, Yunnan Agricultural University, Kunming, Yunnan, China

${ }^{2}$ Kunming Institute of Zoology, Chinese Academy of Sciences, Kunming, Yunnan, China

${ }^{3}$ Animal Husbandry and Veterinary Station of Mangshi City, Mangshi, Yunnan, China

${ }^{4}$ Department of Husbandry and Veterinary, Yunnan Vocational and Technical College of Agriculture,

Kunming, Yunnan, China

*These authors contributed equally to this study.

Corresponding author: Y.W. Miao

E-mail: yongwangmiao999@163.com

Genet. Mol. Res. 13 (2): 3371-3383 (2014)

Received December 10, 2012

Accepted April 30, 2013

Published April 29, 2014

DOI http://dx.doi.org/10.4238/2014.April.29.16

\begin{abstract}
Yunnan is situated in the Southwest China and encompasses regions having high biodiversity, including habitats for several ancestral species of domestic animals such as chicken. Domestic chickens in Yunnan were kept by peoples of varied ethnic and economic backgrounds living in highly varied geographic environments. To identify the genetic background of Yunnan domestic chickens and their relationships with Red Junglefowl, we applied 28 widely used microsatellite DNA markers to genotype 340 birds from 7 chicken breeds and Red Junglefowl indigenous to Yunnan. Among a total of 342 alleles identified, 121 (35.4\%) were breed specific,
\end{abstract}


with Red Junglefowl harboring most microsatellite alleles (23). High levels of heterozygosity were observed within populations indicated by a mean unbiased $H_{\mathrm{E}}$ value of 0.663 , which was higher than the reported for most populations elsewhere. The $F_{\text {IS }}$ value of domestic populations ranged from $-0.098-0.005$, indicating a lack of inbreeding among these populations. A high proportion of significant departures (89) from the $224 \mathrm{HWE}$ tests for each locus in each population reflected an excess of heterozygosity and population substructure. Individual assignment tests, high $F_{\mathrm{ST}}$ values (0.1757-0.3015), and Nei's $\mathrm{D}_{\mathrm{A}}$ genetic distances $(0.4232-0.6950)$ indicated clear differentiation among these populations. These observations, along with the close genetic distance between indigenous domestic populations and Red Junglefowl, were consistent with the primitive and ancestral state of Yunnan indigenous chickens. Protecting the unique variants of these indigenous poultry varieties from contamination with commercial breeds might provide values for improving modern agricultural livestock and breeding programs. Thus, the current study may benefit breeding management and conservation efforts.

Key words: Genetic diversity; Yunnan; Chicken; Red Junglefowl; Microsatellite DNA

\section{INTRODUCTION}

Yunnan is situated in Southwest China at $21^{\circ} 8^{\prime} 32^{\prime \prime}-29^{\circ} 15^{\prime} 8^{\prime \prime} \mathrm{N}$ and $97^{\circ} 31^{\prime} 39^{\prime \prime}-106^{\circ} 11^{\prime} 47^{\prime \prime} \mathrm{E}$, including the southern part of the Qinghai-Tibet Plateau, and the northern edge of tropical Southeast Asia near the Indian and Pacific Oceans with the Tropic of Cancer running through its southern parts. Yunnan neighbors Sichuan in the north and Tibet in the northwest, borders Myanmar in the west and southwest, and is bounded by Laos and Vietnam to the south. The general terrain of Yunnan province falls gradually from the northwest to southeast. Yunnan's highly diverse geography comprises a network of many mountains, rivers, valleys, and lakes, and a plateau alternating with basins, low hills, subalpine mountains, and alpine regions. The climate of Yunnan is dominated by dry continental monsoon in winters and humid oceanic monsoon in summers. Its complex topography divides Yunnan into 7 climate subtypes and up to 30 different ecosystems.

With these highly diversified geographical environments, coupled with copious precipitation, adequate sunlight, and long frost-free periods, Yunnan represents a key terrestrial biodiversity center of global significance. It is home to over half of China's total species of higher plant and vertebrate species and many species of rare, endemic, and epibiotic wildlife. Moreover, because of its complex topography and richness in biodiversity, people's habitats in Yunnan are often isolated from each other. As a result, many diverse ethnic tribes have evolved that account for half of all ethnic tribes in China. Yunnan is also the habitat for wild ancestors of several species of domestic animals and has been suggested to be a center of domestication of common domestic animals such as dog, pig, and chicken (Liu et al., 2006; Wu et al., 2007; Pang et al., 2009). The domestic chickens in Yunnan are raised in highly varied geographical environments by people with varied ethnic backgrounds and in diverse economic conditions. Accordingly, Yunnan's chickens can be regarded as vestiges of the ancestral populations of domestic chicken. Therefore, determining the genetic background of Yunnan domestic chicken 
varieties and their relationship with local Red Junglefowl could provide an improved understanding of chicken evolution and dispersal during its domestication and enable the evaluation of chicken genetic diversity and strategies for conserving its ancestral varieties. Although some local Yunnan chicken breeds have been included in genetic studies of chicken breeds using microsatellite DNA, only a few studies have discussed the diversity of Yunnan local populations in the context of their ancestral state and relationship with Red Junglefowl in remaining habitats of wild chicken populations (Qu et al., 2006; Bao et al., 2008; Li et al., 2009).

In this study, we have used 28 microsatellite markers located on 21 chromosomes or linkage groups to investigate the genetic variability, population structure, and genetic relationships within 7 local chicken breeds (i.e., Banna Gamecock, Chahua chicken, Nixi chicken, Tengchong snow chicken, Wuding chicken, Xichou black-bone chicken, Yanjin black-bone chicken) and Red Junglefowl (Gallus gallus spadiceus) in Yunnan, China.

\section{MATERIAL AND METHODS}

\section{Domestic chicken and Red Junglefowl populations}

In total, 310 chicken blood samples were collected from remote villages representing the original habitat of local chicken breeds. Blood samples of 30 Red Junglefowls were also collected by the Yunnan Humane Society for Wild Animals in Dehong region, Yunnan province. Distinct sexual dimorphism was observed in all breeds and in Red Junglefowl. The habitat environments and morphological characterization of sampled populations are described below (Figure 1).

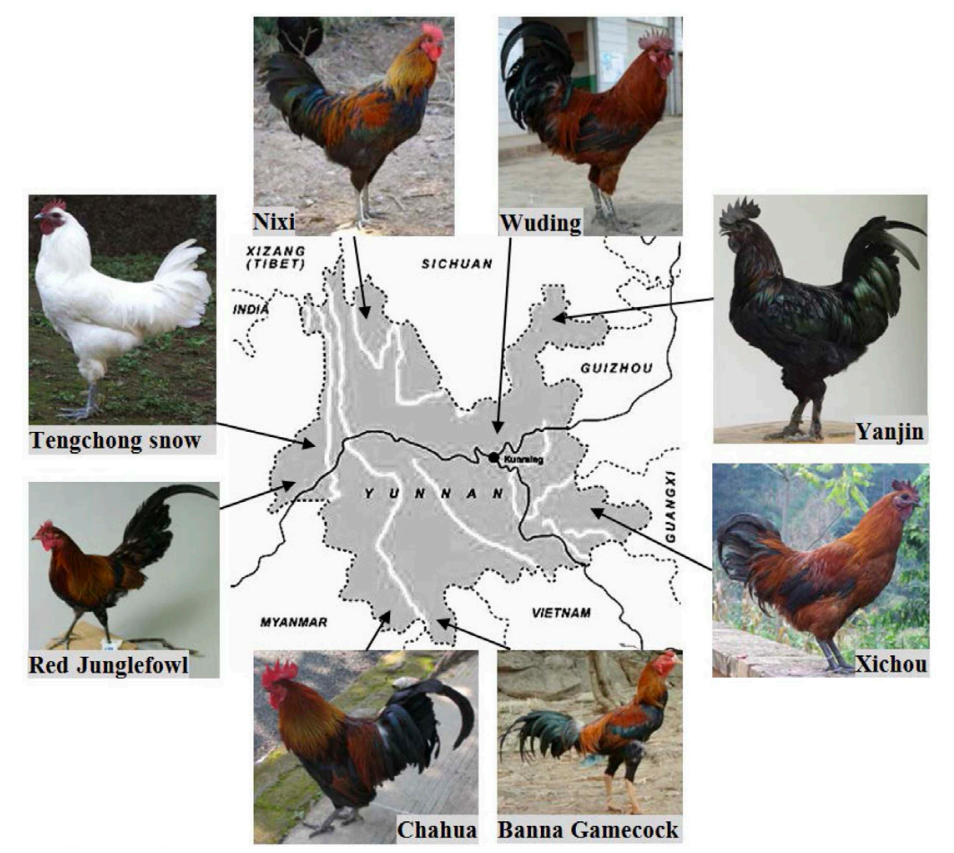

Figure 1. Typical males of Yunnan local chicken breeds and Red Junglefowl. The morphology for each breed and Red Junglefowl was described in the text. 
Banna Gamecock is an entertainment-type breed with a large and muscular body, naked neck and chest, small red earlobe and walnut comb, long neck, and white skin. Plumage color is versatile but is mostly golden red mixed with grayish-green. The body weight in adults is about $2000 \mathrm{~g}$ for males and $1400 \mathrm{~g}$ for females (Luo et al., 1987). Banna Gamecock habitats are located in southwest Yunnan at an elevation of approximately 500-1000 m, characterized by tropical and subtropical climate and representing a major part of the province that houses the richest wildlife resources.

Chahua chicken is assumed to be a primitive type of chicken breed with red or yellow saddle hackles, a black-green tail feathers, red wattle, black shank and beak, single red comb, and mixed grayish-green and yellow plumage tightly sticking to the skin. Chahua chicken is vigilant and good at flying and running. They have a small muscular and small body. Body weights of adults are about $1190 \mathrm{~g}$ for males and $1000 \mathrm{~g}$ for females (Luo et al., 1987). Chahua chicken habitats are located in southwestern and western Yunnan.

Nixi chicken is reared for egg production, yielding 150-180 eggs per year. This breed has a small body size with adults reaching weights of about $1400 \mathrm{~g}$ (males) and 1200 $\mathrm{g}$ (females) (Luo et al., 1987). Nixi chicken has compact plumage, red single comb, an upright black-green and long-tail feather, white skin, and gray shank. The color of its plumage is variable. Nixi chicken habitats are located in northwestern Yunnan, belonging to the Qinghai-Tibet Plateau with high snow-capped mountains and deep valleys formed by the Nujiang River, the Lantsang River, and the upper reaches of the Yangtze River. Subtropical xerothermic valleys, temperate mountainsides, and cold tops contribute to a complex vertical environment.

Tengchong snow chicken is reared for both meat and eggs, and features a red single comb white plumage and tail. Most Tengchong snow chickens have black skin, shanks, muscle, and bone with an adult weight of about $2100 \mathrm{~g}$ (males) and $1600 \mathrm{~g}$ (females). Tengchong snow chicken habitats are located in western Yunnan, which is characterized by deep valleys surrounding the Gaoligongshan Mountain with precipitous terrain and great differences in altitude.

Wuding chicken is a dual-type breed for production of meat and eggs. This breed consists of 2 types having a small or large body size, and it has thick and puffy plumages of diverse color, black tail feathers, single red comb, gray beak and leg, and varied skin and bone color. Some Wuding chickens have feathered toes. The body weight in adults is 3050 $\mathrm{g}$ for the large type and $2100 \mathrm{~g}$ for the small type (Luo et al., 1987). Its habitat is the basin area of the central Yunnan plateau with an average elevation of $1700 \mathrm{~m}$ and belonging to a northern region with subtropical climate. However, the sub-climate and topography within this region is highly variable because of plateaus alternating with basins, low hills, and subalpine mountains.

Xichou black-bone chicken is another dual-type breed. It is vigilant and aggressive and has black skin, shank and bone, a diverse plumage color, red-black comb, wattle, earlobe, and beak. The body weight of mature chickens is $2030 \mathrm{~g}$ (males) and $1770 \mathrm{~g}$ (females). Xichou black-bone chicken habitats are located in southeastern Yunnan, and are characterized by karst topography with a crisscrossing network of numerous mountains, rivers, valleys, and lakes.

Yanjin black-bone chicken is reared for meat by local people. The chicken has a large body size with an adult body weight of $3180 \mathrm{~g}$ (males) and $2200 \mathrm{~g}$ (females), variable plumage color (mainly black and white), upright neck, green-black tail feathers, black skin, beak, 
comb, wattle, ear, and face (Luo et al., 1987). Yanjin black-bone chicken habitats are located in northeast Yunnan, characterized by steep landforms with slopes and bare topsoil, cut by deep valleys and an average elevation of about $1000 \mathrm{~m}$.

Red Junglefowl (Gallus gallus spadiceus, GGS) displays a very strong sexual dimorphism. The female of this species is substantially smaller than the male. The males of G. gallus spadiceus have large red wattles, bright gold and bronze plumage, and up-right long bronzeblack tail feathers. The female's plumage is highly variable and designed for camouflage. Red Junglefowl is similar in many ways to today's domestic chicken. The Red Junglefowl sampled in this study were from a habitat located in western Yunnan.

\section{DNA extraction and microsatellite genotyping}

Blood samples were taken from the ulnar vein and stored at $-40^{\circ} \mathrm{C}$. Genomic DNA was isolated from whole blood of chicken by standard phenol/chloroform extraction methods.

Twenty-eight highly polymorphic microsatellite DNA markers were selected from frequently used microsatellite markers used in previous studies (Wimmers et al., 2000; Shahbazi et al., 2007; Tadano et al., 2007a,b; Tadano et al., 2008; Berthouly et al., 2009). The 28 microsatellite markers covered the autosomes 1-4, 6-13, 15, 17-18, 23, 26-28, and E47W24 and the sex chromosome $\mathrm{Z}$. This selection allowed comparisons of multiple breeds and enabled maximal coverage of the whole chicken genome to minimize physical linkage between the examined loci.

PCR was performed in $25 \mu \mathrm{L}$ reactions containing $50 \mathrm{ng}$ of genomic DNA, 1.5-2.5 $\mathrm{mM} \mathrm{Mg}{ }^{2+}, 2 \mu \mathrm{L}$ 10X buffer, $200 \mu \mathrm{M}$ dNTPs, $1.25 \mathrm{U}$ Taq polymerase (TaKaRa), and $0.4 \mu \mathrm{M}$ of each primer; reactions were cycled under the following conditions: pre-denature at $95^{\circ} \mathrm{C}$ for $10 \mathrm{~min}$, followed by 35 cycles of denaturation at $94^{\circ} \mathrm{C}$ for $1 \mathrm{~min}$, annealing at $50^{\circ}-60^{\circ} \mathrm{C}$ (varied according to loci targeted for amplification) for $1 \mathrm{~min}$, elongation at $72^{\circ} \mathrm{C}$ for $1 \mathrm{~min}$, and a final incubation at $72^{\circ} \mathrm{C}$ for $10 \mathrm{~min}$.

Forward primers were end-labelled with fluorescent dyes (6-FAM). PCR products were genotyped using ABI 3730 DNA Sequencer (Applied Biosystems) with the internal size standard GeneScan $^{\mathrm{TM}} 500$ TAMRA $^{\mathrm{TM}}$ Size Standard (Applied Biosystems).

\section{Statistical analysis}

The GENALEX 6 software (Rod and Smouse, 2006) was used to measure allele diversity, including the number of alleles detected and $F_{\mathrm{ST}}$ value across populations at each locus; within-breed diversity, represented by mean number of alleles (MNA), private alleles, observed heterozygosity $\left(H_{\mathrm{O}}\right)$, and the unbiased expected heterozygosity $\left(\mathrm{UH}_{\mathrm{E}}\right)$ averaged across loci for each population; and to test Hardy-Weinberg equilibrium (HWE) for each breed at each locus. The expected heterozygosity $\left(H_{\mathrm{E}}\right)$ for each microsatellite locus was calculated with the EXCELL TOOLKIT version 3.1.1 (Park, 2001).

The inbreeding coefficients $F_{\text {IS }}$ for each breed and pairwise $F_{\text {ST }}$ values between all chicken breed pairs and Red Junglefowl were calculated according to Weir and Cockerham (1984) using the program FSTAT version 2.9.3.2 (http://www2.unil.ch/popgen/softwares/ fstat.htm). The significance levels for $F_{\mathrm{ST}}$ between all pairs were assessed by sequential Bonferroni correction (Rice, 1989). 
To assess whether any individual could be assigned to or excluded from its own breed or the others, we used the Bayesian exclusion method (Rannala and Mountain, 1997) and the modified Cavalli-Sforza chord distance among individual animals (Nei et al., 1983) imbedded in GENCLASS 2 (Piry et al., 2004). An individual was excluded from a breed if the assignment to this breed had a $\mathrm{P}<0.01$.

Individual clustering was performed by calculating the natural logarithm of the proportion of shared alleles (Bowcock et al., 1994) using Microsatellite Analyzer (MSA) 4.05 (Dieringer and Schlötterer, 2003). A neighbor-joining (NJ) tree of individual clustering based on the proportion of shared alleles (Dps) was constructed with PHYLIP 3.68 (Felsenstein, 2005) and edited using MEGA 4.0 (Tamura et al., 2007).

The genetic relationships among populations were estimated by Nei's $D_{A}$ distance (Nei et al., 1983) using DISPAN (Ota, 1993). A NJ tree for populations based on $\mathrm{D}_{\mathrm{A}}$ distance was constructed using DISPAN (Ota, 1993) with 1000 bootstrap replications and the tree edited in Treeview (Page, 1996) and MEGA 4.0 (Tamura et al., 2007).

\section{RESULTS}

\section{Genetic variation within and between populations}

Among the 8 chicken populations genotyped, all 28 microsatellite loci were polymorphic and in total 342 alleles were found. Across all populations, the average number of alleles per locus was 12.21 (342/28), with an allele range of 5 (MCW0134, MCW0198, MCW0104) to 21 (ADL0284, MCW0005) (Table 1). Of all 342 alleles, 121 (35.4\%) were breed specific, representing unique alleles. Ten of the unique alleles were detected only once, while 47 unique alleles (38.8\%) showed a frequency of $>20 \%$ in the breed. We eliminated unique alleles that had extremely low frequencies because such alleles might have been artifacts. The $H_{\mathrm{E}}$ per locus ranged from 0.635 (MCW0252) to 0.930 (ADL0190), and the $F_{\mathrm{ST}}$ per locus from 0.097 (MCW0119) to 0.348 (MCW0294). As shown in Table 1, all loci displayed a high degree of genetic diversity with a mean of expected heterozygosity across all loci of 0.834 . In general, high genetic differentiation across all loci was found with an average $F_{\text {ST }}$ of 0.215 , which indicated that $78.5 \%$ of the genetic variation among chickens was caused by genetic differences among individuals and $21.5 \%$ was due to differences among populations.

Table 2 shows genetic diversity within each population. The mean number of alleles (MNA) per population ranged from 3.57 (Banna Gamecock) to 4.32 (Wuding chicken, Yanjin black-bone chicken). The number of unique alleles ranged from 9 (Yanjin Black-bone chicken) to 23 (Red Junglefowl). High observed heterozygosity $\left(H_{\mathrm{O}}\right)$ was observed to range from 0.640 (Red Junglefowl) to 0.694 (Tengchong snow), and unbiased expected heterozygosity $\left(\mathrm{UH}_{\mathrm{E}}\right)$ from 0.630 (Nixi chicken) to 0.685 (Tengchong snow). $F_{\text {IS }}$ in domestic populations ranged from -0.098-0.005, indicating lack of inbreeding within the populations, whereas the $F_{\text {IS }}$ value of Red Junglefowl was $0.041(\mathrm{P}<0.05)$, indicating scarcity of heterozygotes in the population of Red Junglefowl (Table 2).

A total of $224 \mathrm{HWE}$ tests were carried out for all 28 polymorphic loci in the 8 populations. Of these 224 tests, 89 showed significant departure from HWE, with the number of loci deviating from the HWE $(\mathrm{P}<0.05)$ in a single test population ranging from 5 (Banna Gamecock) to 17 (Yanjin black-bone chicken). 
Table 1. Characterization of 28 microsatellites analyzed in Yunnan local chicken breeds and Red Junglefowl.

\begin{tabular}{|c|c|c|c|c|c|}
\hline Locus & $\mathrm{N}^{\mathrm{a}}$ & $\mathrm{Na}^{\mathrm{b}}$ & Allele size range (bp) & $H_{\mathrm{E}}{ }^{\mathrm{c}}$ & $F_{\mathrm{ST}}{ }^{\mathrm{d}}$ \\
\hline ADL0251 & 338 & 13 & $200-350$ & 0.861 & 0.281 \\
\hline ADL0188 & 334 & 7 & $150-209$ & 0.683 & 0.113 \\
\hline ADL0257 & 338 & 17 & $162-218$ & 0.911 & 0.184 \\
\hline ADL0190 & 340 & 19 & $205-231$ & 0.930 & 0.289 \\
\hline MCW0252 & 322 & 10 & $281-301$ & 0.635 & 0.257 \\
\hline MCW0224 & 337 & 18 & 291-301 & 0.900 & 0.319 \\
\hline MCW0170 & 328 & 10 & $263-267$ & 0.860 & 0.143 \\
\hline MCW0005 & 340 & 21 & $189-259$ & 0.925 & 0.207 \\
\hline MCW0240 & 310 & 17 & $169-195$ & 0.916 & 0.192 \\
\hline MCW0176 & 298 & 12 & $257-270$ & 0.881 & 0.162 \\
\hline MCW0120 & 333 & 12 & $250-287$ & 0.829 & 0.150 \\
\hline ADL0121 & 335 & 12 & $125-157$ & 0.886 & 0.286 \\
\hline MCW0134 & 333 & 5 & $252-284$ & 0.644 & 0.297 \\
\hline MCW0035 & 337 & 7 & $227-233$ & 0.753 & 0.215 \\
\hline MCW0097 & 340 & 8 & 263-309 & 0.750 & 0.141 \\
\hline MCW0198 & 327 & 5 & $294-324$ & 0.794 & 0.220 \\
\hline MCW0104 & 338 & 5 & $186-263$ & 0.686 & 0.117 \\
\hline MCW0080 & 338 & 12 & $264-337$ & 0.894 & 0.271 \\
\hline MCW0330 & 338 & 13 & $254-290$ & 0.856 & 0.194 \\
\hline MCW0217 & 325 & 11 & $150-174$ & 0.865 & 0.211 \\
\hline MCW0165 & 339 & 8 & $112-144$ & 0.764 & 0.195 \\
\hline MCW0285 & 335 & 9 & $156-300$ & 0.856 & 0.188 \\
\hline MCW0328 & 337 & 15 & $262-324$ & 0.918 & 0.242 \\
\hline ADL0284 & 329 & 21 & $119-167$ & 0.910 & 0.145 \\
\hline LEI0075 & 340 & 17 & $226-259$ & 0.911 & 0.268 \\
\hline MCW0294 & 335 & 18 & $306-317$ & 0.919 & 0.348 \\
\hline MCW0154 & 339 & 14 & $171-193$ & 0.885 & 0.294 \\
\hline MCW0119 & 329 & 6 & $116-180$ & 0.726 & 0.097 \\
\hline All loci & & 342 & - & $0.834 \pm 0.017$ & $0.215 \pm 0.013$ \\
\hline
\end{tabular}

a Total number of individuals genotyped. ${ }^{\mathrm{b}}$ Number of alleles detected across populations. ${ }^{\mathrm{c}} H_{\mathrm{E}}=$ Expected heterozygosity. ${ }^{\mathrm{d}}$ Population differentiation index.

Table 2. Genetic variations of Yunnan local chicken breeds and Red Junglefowl estimated from 28 microsatellite genotypes.

\begin{tabular}{|c|c|c|c|c|c|c|c|c|}
\hline Population ID & $\begin{array}{l}\text { Sample } \\
\text { size }\end{array}$ & $\mathrm{MNA}^{\mathrm{a}} \pm \mathrm{SE}$ & $\begin{array}{c}\text { Private alleles } \\
\quad(>5 \%)\end{array}$ & $H_{\mathrm{O}}{ }^{\mathrm{c}} \pm \mathrm{SE}$ & $H_{\mathrm{E}}^{\mathrm{d}}$ & $\mathrm{UH}_{\mathrm{E}}{ }^{\mathrm{e}} \pm \mathrm{SE}$ & $F_{\text {IS }}{ }^{\mathrm{f}} \pm \mathrm{SE}$ & DWHE $^{g}$ \\
\hline Banna Gamecock & 27 & $3.57 \pm 0.19$ & 11 & $0.656 \pm 0.037$ & $0.625 \pm 0.019$ & $0.637 \pm 0.019$ & -0.031 & 5 \\
\hline Chahua chicken & 31 & $4.14 \pm 0.26$ & 14 & $0.691 \pm 0.034$ & $0.647 \pm 0.023$ & $0.658 \pm 0.024$ & -0.052 & 10 \\
\hline Nixi chicken & 45 & $3.61 \pm 0.23$ & 15 & $0.692 \pm 0.035$ & $0.623 \pm 0.021$ & $0.630 \pm 0.024$ & -0.098 & 14 \\
\hline Tengchong snow & 53 & $4.21 \pm 0.24$ & 10 & $0.694 \pm 0.031$ & $0.678 \pm 0.019$ & $0.685 \pm 0.019$ & -0.014 & 13 \\
\hline Wuding chicken & 53 & $4.32 \pm 0.27$ & 11 & $0.692 \pm 0.034$ & $0.674 \pm 0.024$ & $0.680 \pm 0.024$ & -0.018 & 12 \\
\hline Xichou black-bone & 48 & $4.14 \pm 0.22$ & 12 & $0.678 \pm 0.022$ & $0.673 \pm 0.020$ & $0.681 \pm 0.021$ & 0.005 & 12 \\
\hline Yanjin black-bone & 53 & $4.32 \pm 0.24$ & 9 & $0.680 \pm 0.042$ & $0.658 \pm 0.030$ & $0.665 \pm 0.030$ & -0.024 & 17 \\
\hline Red Junglefowl & 30 & $4.07 \pm 0.16$ & 23 & $0.640 \pm 0.022$ & $0.656 \pm 0.018$ & $0.667 \pm 0.019$ & $0.041^{*}$ & 6 \\
\hline
\end{tabular}

${ }^{\mathrm{a}}$ Mean number of alleles per locus. ${ }^{\mathrm{b}}$ Number of private alleles with frequency $>5 \%$. ${ }^{\mathrm{c}}$ Observed heterozygosity, average across loci. ${ }^{\mathrm{d}}$ Expected heterozygosity, average across loci. ${ }^{\mathrm{e}} \mathrm{UHe}=$ unbiased expected heterozygosity, average across loci. Inbreeding index for each breed. ${ }^{\mathrm{f}}$ Number of loci that deviated from Hardy-Weinberg equilibrium $(\mathrm{P}<0.05)$. $* \mathrm{P}<0.05$.

\section{Population differentiation}

By using both Bayesian inference and Nei’s Dps distance at the 5\% level, we per- 
formed individual clustering of 340 microsatellite data samples, which successfully assigned all individuals into their original populations on the basis of 28 loci genotypes. A NJ tree based on Dps (Figure 2) showed that all individuals were correctly placed into the population from which they originated.

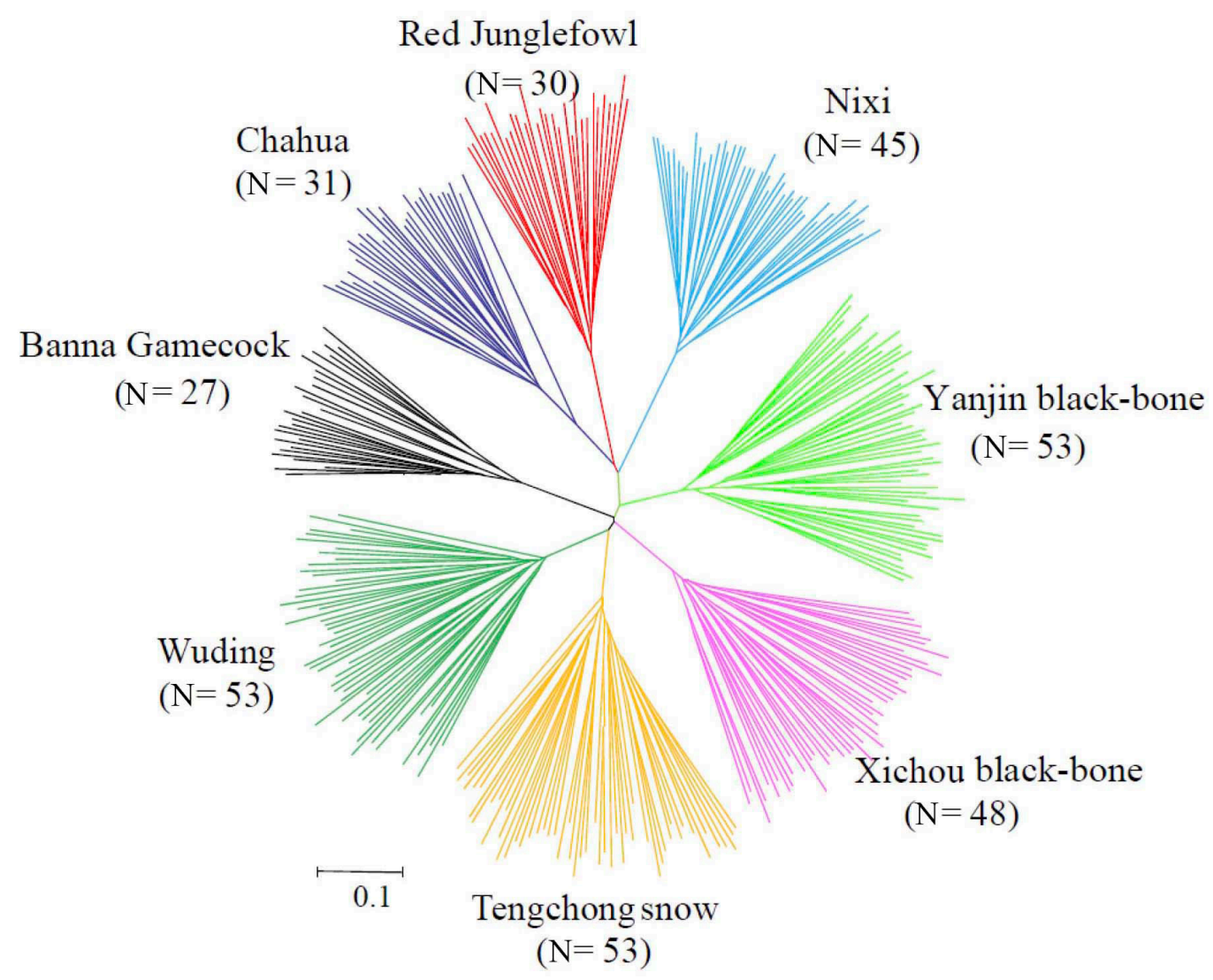

Figure 2. Unrooted neighbor-joining tree summarizing genetic relationships among 340 birds from Yunnan chicken breeds and Red Junglefowl. Distance was measured using the natural logarithm of the proportion of shared alleles (Dps) calculated from 28 microsatellite loci.

The results for the calculated population differentiation index $F_{\mathrm{ST}}$ and Nei's $\mathrm{D}_{\mathrm{A}}$ distance between pairs of populations are shown in Table 3. Large genetic differentiation $\left(F_{\mathrm{ST}}\right)$ was observed between all population pairs $(\mathrm{P}<0.01)$. The highest $F_{\mathrm{ST}}$ was observed between the breeds Banna Gamecock and Nixi (0.3015), and the lowest $F_{\mathrm{ST}}$ was observed between Wuding and Tengchong snow (0.1757). Accordingly, the highest and lowest Nei's $\mathrm{D}_{\mathrm{A}}$ distance was between Banna Gamecock and Nixi (0.6950) and between Wuding and Tengchong snow (0.4232), respectively. The NJ tree based on Nei's $\mathrm{D}_{\mathrm{A}}$ showed clear separations between the populations indicated by long branch lengths (Figure 3). Red Junglefowl, Chahua, and Nixi clustered together, while the remaining breeds formed the other major clade. 
Table 3. Nei's $\mathrm{D}_{\mathrm{A}}$ genetic distance (below diagonal) and population differentiation $F_{\mathrm{ST}}$ value (above diagonal) between pairs of chicken breeds.

\begin{tabular}{|c|c|c|c|c|c|c|c|c|}
\hline & $\begin{array}{c}\text { Banna } \\
\text { Gamecock }\end{array}$ & Chahua & Nixi & $\begin{array}{l}\text { Tengchong } \\
\text { snow }\end{array}$ & Wuding & $\begin{array}{c}\text { Xichou } \\
\text { Black-bone }\end{array}$ & $\begin{array}{c}\text { Yanjin } \\
\text { Black-bone }\end{array}$ & $\begin{array}{c}\text { Red } \\
\text { Junglefowl }\end{array}$ \\
\hline Banna Gamecock & & $0.2416^{* *}$ & $0.3015 * *$ & $0.2185^{* *}$ & $0.2087 * *$ & $0.2181 * *$ & $0.2193^{* *}$ & $0.2590^{* *}$ \\
\hline Chahua & 0.5709 & & $0.2587 * *$ & $0.2323 * *$ & $0.2414 * *$ & $0.2412 * *$ & $0.2276^{* *}$ & $0.2160 * *$ \\
\hline Nixi & 0.6950 & 0.5995 & & $0.2592 * *$ & $0.2596^{* *}$ & $0.2334 * *$ & $0.2411 * *$ & $0.2716^{* *}$ \\
\hline Tengchong snow & 0.5532 & 0.6155 & 0.6583 & & $0.1757 * *$ & $0.1945^{* *}$ & $0.1899^{* *}$ & $0.2248 * *$ \\
\hline Wuding & 0.4995 & 0.5876 & 0.6169 & 0.4232 & & $0.1826 * *$ & $0.1912^{* *}$ & $0.2556^{* *}$ \\
\hline Xichou Black-bone & 0.5227 & 0.5949 & 0.5895 & 0.4977 & 0.4287 & & $0.1957 * *$ & $0.2515^{* *}$ \\
\hline Yanjin Black-bone & 0.5102 & 0.5049 & 0.5716 & 0.4628 & 0.4558 & 0.4938 & & $0.2489 * *$ \\
\hline Red Junglefowl & 0.6189 & 0.5080 & 0.6500 & 0.6051 & 0.6619 & 0.6625 & 0.5871 & \\
\hline
\end{tabular}

Highest and lowest values are in bold. ${ }^{* *} F_{\mathrm{ST}}$ values are significantly different from zero $(\mathrm{P}<0.01)$.

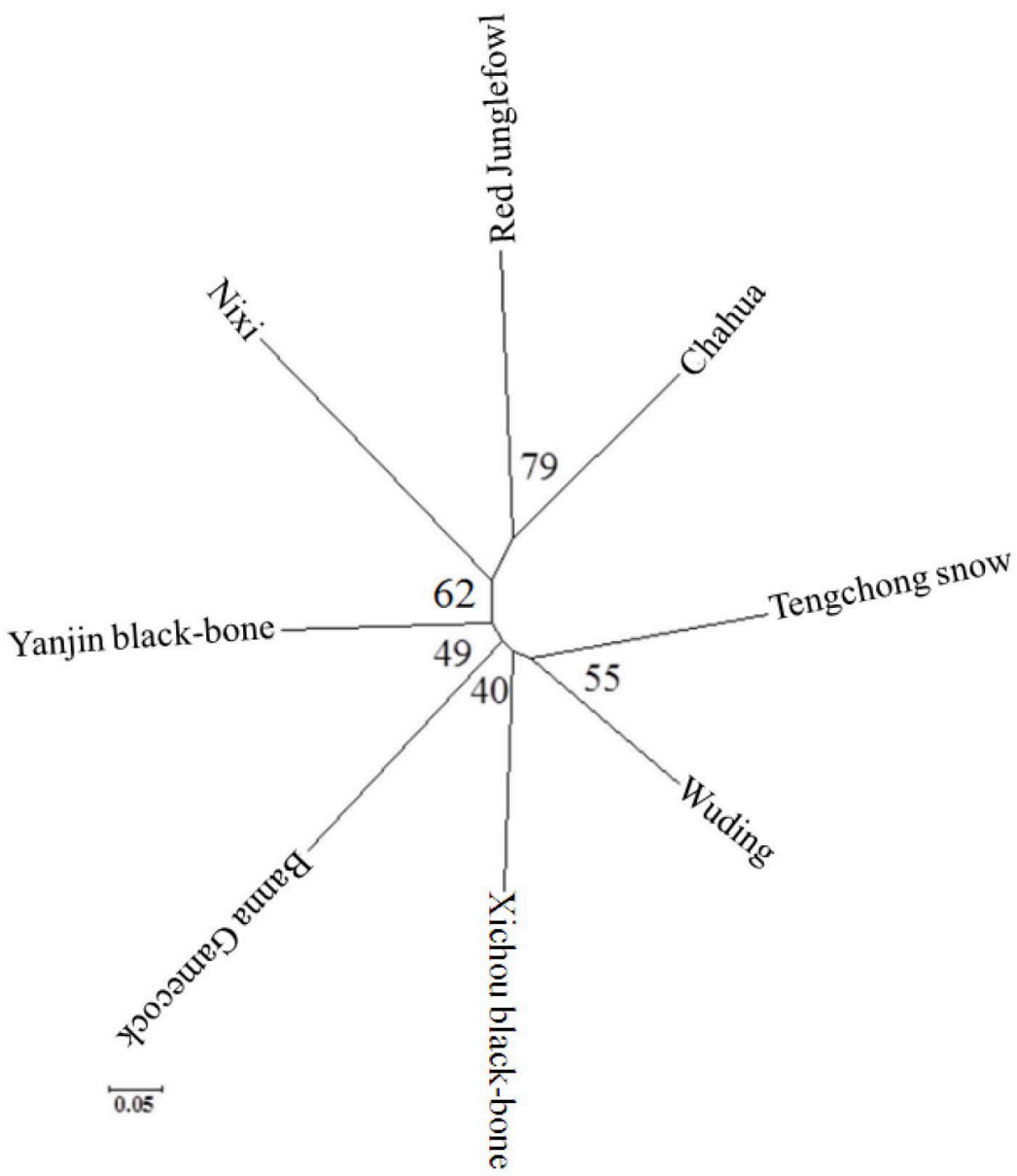

Figure 3. Unrooted neighbor-joining tree shows the genetic relationships among Yunnan local chicken breeds and Red Junglefowl, using Nei's $\mathrm{D}_{\mathrm{A}}$ genetic distance calculated from 28 microsatellite loci. The number at each node represents percentage of bootstrap value from 1000 replications. 


\section{DISCUSSION}

\section{Genetic diversity}

As expected, high heterozygosity was observed in all Yunnan local breeds. The heterozygosity of Wuding and Chahua was higher in this study than reported in previous studies (Qu et al., 2006; Bao et al., 2008). The $H_{\mathrm{F}}$ for Red Junglefowl and local chicken breeds from Thailand and Vietnam was close to that for Red Junglefowl in this study (Berthouly et al., 2009). In general, the heterozygosity of Yunnan local chicken breeds in this study was higher than in most populations from Africa, Europe, and Asia (Crooijmans et al., 1996; Wimmers et al., 2000; Cheng et al., 2003; Qu et al., 2006; Bao et al., 2007; Granevitze et al., 2007; Tadano et al., 2007a,b; Bao et al., 2008; Berthouly et al., 2008; Kaya and Yildiz, 2008; Tadano et al., 2008; Berthouly et al., 2009), but comparable with populations from Iran (Shahbazi et al., 2007) and H'mong chicken from Vietnam (Granevitze et al., 2007). The high genetic diversity of Yunnan local chicken was consistent with their primitive state and the fact that they are the result of breeding methods used by people with diverse ethnic backgrounds living in different economic conditions in complex environments. Thus, Yunnan local chicken represents a unique genetic resource for modern poultry husbandry.

We observed that 89 cases of 224 tested combinations of Yunnan chicken population with microsatellite loci departed from Hardy-Weinberg equilibrium, representing a higher proportion than what has been observed in previous studies (Qu et al., 2006; Bao et al., 2008). The reasons for deviation from HWE vary and include inbreeding or outbreeding, selection, population substructure, and existence of "null alleles". The inbreeding index for all domestic chicken breeds except Xichou black-bone chicken was below zero, which indicates that inbreeding could be excluded as a cause for the high deviation from HWE. The clear differentiation from each breed indicated that outbreeding among local breeds was not likely. The breeding history and the primitive state of Yunnan local chicken indicate that high selection could also be excluded. The "null alleles" for the microsatellite loci analyzed were not reported in previous studies. For complex breeding environments, isolation within a geographic population is possible, and such isolation could lead to substructures within populations. As seen in Figure 2, some subclades were discernible within some breeds, such as Nixi, Yanjin black-bone, and Wuding chicken. So the existence of population substructures with an excess of heterozygosity might be the most likely reason for the high frequency of departure from HWE observed in our study.

The inbreeding coefficient $F_{\text {IS }}$ for Red Junglefowl was positive $(\mathrm{P}<0.05)$. Since the Red Junglefowl population in this study was obtained from Yunnan Humane Society for Wild Animals and has been kept for several generations, the observed $F_{\text {IS }}$ may be due to inbreeding and genetic drift in the Red Junglefowl population studied here.

\section{Population differentiation}

The extremely diverse and complex geographical environments in Yunnan have led to many relatively isolated ecosystems, thus leading to isolated populations of the local animals and people. To assess if clearly differentiated breeds or populations exist in this region, individual clustering was performed. The individual clustering of 340 samples successfully assigned all individuals into their original populations (Figure 2), which demonstrated clear 
genetic differentiation among populations of Yunnan local chicken. Thus, the traditional breed classification based on morphology and usage was well established.

The high genetic distance and $F_{\mathrm{ST}}$ values among populations showed that these populations are genetically highly differentiated (Table 3 ). The NJ tree based on DA distance reflected this genetic differentiation (Figure 3). In the NJ tree, the Red Junglefowl, and Chahua clustered together. The Chahua chicken was distributed in southwestern and western Yunnan, which represents the main habitat of Red Junglefowl. The morphology of Chahua is most closely similar to the local Red Junglefowl. It has been widely accepted that there is gene flow between Chahua and Red Junglefowl. For example, Chahua chicken is used as decoy by local hunters to capture wild Red Junglefowl (Luo et al., 1987). As an egg-producing chicken, Nixi chicken resides in northwestern Yunnan, belonging to the Qinghai-Tibet Plateau with high snow-capped mountains and valleys deeply cut by the Nujiang River, the Lantsang River, and the upper reaches of the Yangtze River. The habitat of Nixi is one of the most inaccessible regions in Yunnan. So this habitat and the use of Nixi as an egg producer explains well that the genetic distances between Nixi and the other dual-purpose populations were among the largest, consistent with a previous study (Zhang et al., 1997). The genetic distances between Wuding and other populations were the smallest (Table 3), which might due to the habitat of Wuding chicken being located in the central part of Yunnan. Most chickens in the central region of Yunnan and its vicinities share multiple characteristics, including black skin, shanks, muscle, and bones, indicating that these chicken breeds share some common genes from recent ancestors. These populations are mainly raised for meat use. The topographic environment of these regions consists of relatively low terrain more amenable to human habitation. Therefore, besides being derived from a recent ancestral population, gene flow among these chicken populations may have been enabled by more frequent interactions between the people living in these regions.

The genetic distances between Red Junglefowl and the domestic breeds were not as high as has been observed in other studies (Bao et al., 2008; Tadano et al., 2008). This might be due to the reduced heterozygosity of Red Junglefowl cause by genetic drift. Alternatively, since the Yunnan local chicken represents the primitive breeds and cohabitated with local Red Junglefowl, these genetic relationships might also reflect the particular phylogenetic relationship of the Red Junglefowl with Yunnan local domestic chickens.

With its extremely diverse and complex geographical environments, Yunnan harbors the wild ancestors of several kinds of domestic animals and is regarded as the domestication center for several common domestic animals, such as dog, pig, and chicken (Liu et al., 2006; Wu et al., 2007; Pang et al., 2009). Microsatellite variations revealed in this study confirmed the primitive state of Yunnan indigenous chickens as ancestral populations of chicken. Protecting the unique variants of these primitive chicken varieties from contamination with commercial breeds might of particular value for improving modern agricultural livestock and breeding programs. Thus, the current study may benefit and inform breeding management and conservation efforts.

\section{ACKNOWLEDGMENTS}

Research supported by the Science Foundation of the Educational Commission of Yunnan Province of China (\#5Y0196B) and the National High Technology Research and Development Program of China ("863” Program, \#2011AA100305). 


\section{REFERENCES}

Bao W, Chen G, Li B, Wu X, et al. (2008). Analysis of genetic diversity and phylogenetic relationships among red jungle fowls and Chinese domestic fowls. Sci. China C Life Sci. 51: 560-568.

Bao WB, Chen GH, Wu XS, Xu Q, et al. (2007). Genetic diversity of red jungle fowl in China (Gallus gallus spadiceus) and red jungle fowl (Gallus gallus gallus) in Thailand. Yi Chuan 29: 587-592.

Berthouly C, Bed'Hom B, Tixier-Boichard M, Chen CF, et al. (2008). Using molecular markers and multivariate methods to study the genetic diversity of local European and Asian chicken breeds. Anim. Genet. 39: 121-129.

Berthouly C, Leroy G, Van TN, Thanh HH, et al. (2009). Genetic analysis of local Vietnamese chickens provides evidence of gene flow from wild to domestic populations. BMC Genet. 10: 1.

Bowcock AM, Ruiz-Linares A, Tomfohrde J, Minch E, et al. (1994). High resolution of human evolutionary trees with polymorphic microsatellites. Nature 368: 455-457.

Cheng HJ, Yue YS, Fan XZ, Zhang CS, et al. (2003). Analysis of genetic diversity of Shandong indigenous chicken breeds using microsatellite marker. Yi Chuan Xue Bao 30: 855-860.

Crooijmans RP, Groen AF, Van Kampen AJ, Van der Beek S, et al. (1996). Microsatellite polymorphism in commercial broiler and layer lines estimated using pooled blood samples. Poult. Sci. 75: 904-909.

Dieringer D and Schlötterer C (2003). Microsatellite analyser (msa): A platform independent analysis tool for large microsatellite data sets. Mol. Ecol. Notes 3: 167-169.

Felsenstein J (2005). PHYLIP (Phylogeny Inference Package) Version 3.6 Distributed by the Author. Department of Genome Sciences, University of Washington, Seattle.

Granevitze Z, Hillel J, Chen GH, Cuc NT, et al. (2007). Genetic diversity within chicken populations from different continents and management histories. Anim. Genet. 38: 576-583.

Kaya M and Yildiz MA (2008). Genetic diversity among Turkish native chickens, Denizli and Gerze, estimated by microsatellite markers. Biochem. Genet. 46: 480-491.

Li HF, Han W, Zhu YF, Shu JT, et al. (2009). Analysis of genetic structure and relationship among nine indigenous Chinese chicken populations by the Structure program. J. Genet. 88: 197-203.

Liu YP, Wu GS, Yao YG, Miao YW, et al. (2006). Multiple maternal origins of chickens: out of the Asian jungles. Mol. Phylogenet. Evol. 38: 12-19.

Luo QL, Wen JK, Wang X and Li ZS (1987). The Breeds of Domestic Animals in Yunnan. Yunnan Science and Technology Press, Kunming.

Nei M, Tajima F and Tateno Y (1983). Accuracy of estimated phylogenetic trees from molecular data. II. Gene frequency data. J. Mol. Evol. 19: 153-170.

Ota T (1993). DISPAN: Genetic Distance and Phylogenetic Analysis. Institute of Molecular Evolutionary Genetics, Pennsylvania State University, Pennsylvania, PA.

Page RD (1996). TreeView: an application to display phylogenetic trees on personal computers. Comput. Appl. Biosci. 12: 357-358.

Pang JF, Kluetsch C, Zou XJ, Zhang AB, et al. (2009). mtDNA data indicate a single origin for dogs south of Yangtze River, less than 16,300 years ago, from numerous wolves. Mol. Biol. Evol. 26: 2849-2864.

Park SDE (2001). Trypanotolerance in West African Cattle and the Population Genetic Effects of Selection. ph.D. thesis, University of Dublin, Dublin.

Piry S, Alapetite A, Cornuet JM, Paetkau D, et al. (2004). GENECLASS2: a software for genetic assignment and firstgeneration migrant detection. J. Hered. 95: 536-539.

Qu L, Li X, Xu G, Chen K, et al. (2006). Evaluation of genetic diversity in Chinese indigenous chicken breeds using microsatellite markers. Sci. China C Life Sci. 49: 332-341.

Rannala B and Mountain JL (1997). Detecting immigration by using multilocus genotypes. Proc. Natl. Acad. Sci. U. S. A. 94: 9197-9201.

Rice WR (1989). Analyzing tables of statistical tests. Evolution 43: 223-225.

Rod P and Smouse PE (2006). Genalex 6: Genetic analysis in excel. Population genetic software for teaching and research. Mol. Ecol. Notes. 6: 288-295.

Shahbazi S, Mirhosseini SZ and Romanov MN (2007). Genetic diversity in five Iranian native chicken populations estimated by microsatellite markers. Biochem. Genet. 45: 63-75.

Tadano R, Nishibori M, Nagasaka N and Tsudzuki M (2007a). Assessing genetic diversity and population structure for commercial chicken lines based on forty microsatellite analyses. Poult. Sci. 86: 2301-2308.

Tadano R, Sekino M, Nishibori M and Tsudzuki M (2007b). Microsatellite marker analysis for the genetic relationships among Japanese long-tailed chicken breeds. Poult. Sci. 86: 460-469. 
Tadano R, Nishibori M, Imamura Y, Matsuzaki M, et al. (2008). High genetic divergence in miniature breeds of Japanese native chickens compared to Red Junglefowl, as revealed by microsatellite analysis. Anim. Genet. 39: 71-78.

Tamura K, Dudley J, Nei M and Kumar S (2007). MEGA4: Molecular Evolutionary Genetics Analysis (MEGA) software version 4.0. Mol. Biol. Evol. 24: 1596-1599.

Wimmers K, Ponsuksili S, Hardge T, Valle-Zarate A, et al. (2000). Genetic distinctness of African, Asian and South American local chickens. Anim. Genet. 31: 159-165.

Wu GS, Yao YG, Qu KX, Ding ZL, et al. (2007). Population phylogenomic analysis of mitochondrial DNA in wild boars and domestic pigs revealed multiple domestication events in East Asia. Genome Biol. 8: R245.

Zhang TQ, Zou P, Su B and Liu AH (1997). The genetic relationship of Yunnan indigenous chicken populations based on blood protein polymorphism. Yunnan J. Anim. Sci. Vet. Med. 3: 27-30. 